\title{
iglrestre
}

\section{Uso de mídias digitais como auxílio no ensino de Parasitologia}

\author{
USE OF DIGITAL MEDIA AS AN AID IN PARASITOLOGY TEACHING
}

Ana Carolina Fonseca Lindoso Melo', Reginaldo Almeida da Trindade², Domingos Alves ${ }^{3}$

\author{
1 Doutora em Ciências Veterinárias. Universidade \\ Federal do Ceará. \\ ORCID: https://orcid.org/0000-0003-4869-7882 \\ Email: carolinamelo@ufc.br \\ 2 Doutor em Biotecnologia. Universidade Federal do \\ Rio de Janeiro. \\ ORCID: https://orcid.org/0000-0003-3809-3145 \\ Email: rtrindade@pharma.ufrj.br \\ 3 Doutor em Física. Universidade de São Paulo. \\ ORCID: https://orcid.org/0000-0002-0800-5872 \\ Email: doquiron@gmail.com
}

Correspondência: Rua Monsenhor Furtado, S/N. Departamento de Patologia e Medicina Legal, Faculdade de Medicina. Fortaleza, CE. Brasil. CEP: 60430-160.

\section{Copyright: Esta obra está licenciada com uma Licença Creative Commons Atribuição-NãoComercial 4.0 Internacional.}

Conflito de interesses: os autores declaram que não há conflito de interesses.

\section{Como citar este artigo}

Melo ACFL; Trindade RA da; Alves D. Uso de mídias digitais como auxílio no ensino de Parasitologia. Revista de Saúde Digital e Tecnologias Educacionais. [online], volume 5, n. 2. Editor responsável: Luiz Roberto de Oliveira. Fortaleza, julho de 2020, p. 161-174. Disponível em: $\quad$ http://periodicos.ufc.br/resdite/index. Acesso em "dia/mês/ano".

Data de recebimento do artigo: $15 / 10 / 2020$

Data de aprovação do artigo: 28/06/2020

Data de publicação: $21 / 07 / 2020$

\begin{abstract}
Resumo
Introdução: 0 ensino na área da saúde, geralmente, ocorre na forma tradicional. 0 advento das Tecnologias Digitais de Informação e Comunicação tornou-se um meio de transformação do processo de ensino e aprendizagem. Este estudo descreve as publicações sobre o uso de tecnologias digitais no ensino de Parasitologia. Métodos: Revisão bibliográfica integrativa, realizada nas bases de dados LILACS, SCIELO, Google Academics e Google, de trabalhos publicados entre 2008 a 2019 , em português ou espanhol, no tema ensino de parasitologia com tecnologias digitais. Resultados: Vinte publicações (dezesseis artigos e quatro trabalhos acadêmicos) foram selecionadas. Os públicos alvos foram: alunos do ensino fundamental, profissionais em formação técnica e graduandos em ciências da saúde e biológicas. Três trabalhos apenas expuseram materiais interativos. As tecnologias digitais mais propostas foram: sítios eletrônicos, softwares, plataformas de ensino online, atlas virtuais e jogos digitais. Um trabalho mais recente propôs um aplicativo de celular. Conclusão: 0 uso de tecnologias digitais é uma ferramenta valiosa no ensino de Parasitologia e se apresenta por meio de estratégias diversificadas. Entretanto, considerando as possibilidades de uso de tecnologias digitais em uma disciplina com muitos conceitos visuais, a ampliação das estratégias interativas para ensino de Parasitologia permanece como uma área próspera para novos estudos.
\end{abstract}

Palavras-Chave: Materiais de ensino, Realidade virtual, Parasitologia. 
Introduction: Teaching in the field of health education is commonly performed by means of the traditional methods. The advent of Digital Information and Communication Technologies has become a mean of transforming teaching and learning processes. This study describes publications on the use of digital technologies for teaching Parasitology. Methods: Integrative literature review, carried out in the databases LILACS, SCiELO, Google Academics and Google, of studies published between 2008 and 2019, in Portuguese or Spanish, on the subject of teaching parasitology using digital technologies. Results: Twenty studies (sixteen papers and four academic texts) were selected. The target audiences were: elementary school students, professionals in technical training, teaching trainees and undergraduate students in health and biological sciences courses. Three papers only presented interactive materials. The most planned digital technologies were: sites, software, online platforms, digital games and virtual Atlas. One recent study presented an app for smartphones. Conclusion: The use of digital technologies is a valuable tool for teaching Parasitology. They are presented through diversified strategies. However, considering the multiple possibilities of using digital technologies in a course which has many visual concepts, the expansion of interactive strategies for teaching Parasitology remains a thriving area for further studies.

Keywords: Parasitology, Teaching materials, Virtual reality.

\section{Introdução}

Apesar das muitas mudanças que as tecnologias digitais inseriram no processo ensino e aprendizagem, ainda se constata que há um predomínio da forma tradicional de ensinar e aprender, mesmo no ensino superior e, particularmente, nas áreas da saúde. Sabese que a sala de aula como local de aprendizagem deve ser compreendida como um espaço de compartilhamento de saberes singulares provenientes tanto de professores como de estudantes. Entretanto, na prática do dia a dia, ainda prevalece o protagonismo do professor, na perspectiva do detentor do conhecimento, ignorando-se a possibilidade de também propiciar momentos de protagonismo ativo dos aprendizes ${ }^{1}$. O avanço tecnológico e, com ele, as novas modalidades de compartilhamento do conhecimento se tornaram muito afins a esta nova geração de estudantes que nasceram e convivem com maior facilidade em meio às tecnologias. Sob a perspectiva do aprendizado, essas mudanças têm impactado o processo educativo em muitas dimensões, ou seja, nos espaços e ambientes de aprendizagem, nos recursos e metodologias de ensino, na participação do professor e, especialmente, no perfil de estudantes dessa nova geração.

Para Palácio e Strunchiner ${ }^{1}$, o uso das tecnologias digitais de informação e comunicação (TDICs) no ensino de graduação nas ciências da saúde é uma importante estratégia que vem permitindo a transformação da prática educativa tradicional. Nesse caso, entende-se por prática educativa tradicional a ação de ensino verticalizada e centrada na figura do professor como único detentor e transmissor do conhecimento ${ }^{2}$. É justamente nessa mudança de estratégia que a aplicação das TDICs tem mais a contribuir, pois sua 
implementação nos diversos níveis da educação (fundamental, técnico e superior) inicia com a exigência de uma proposta pedagógica que valorize a participação ativa (interatividade) de todos os atores envolvidos e propicie um movimento dialógico real no processo de ensino e aprendizagem ${ }^{1}$. Processo esse que ocorre tanto nos modelos de ensino formal e tradicional (entendido nesse ponto como o processo de ensino e aprendizagem que ocorre exclusivamente no espaço de sala de aula), quanto informal (entendido aqui como o processo de ensino que pode ocorrer em qualquer espaço não exclusivo à sala de aula), sendo verificado espaço para o emprego das mais diversificadas ferramentas digitais no processo de construção do conhecimento ${ }^{3}$.

Como o uso das TDICs vem se firmando cada vez mais na educação, diversos autores têm desenvolvido materiais digitais de apoio que se apresentam como importantes ferramentas no processo de aprendizagem. Esses materiais contribuem para o melhor entendimento e para a fixação do conteúdo ministrado, preenchendo lacunas deixadas pelo método de ensino tradicional. Em complementação às metodologias clássicas, as TDICs consolidam-se como novas ferramentas no processo de ensino e aprendizagem, especialmente na área da Parasitologia ${ }^{4,5,6}$. A Parasitologia é uma área da biologia e da saúde cujo conteúdo ministrado trabalha com muitos esquemas e conceitos para entendimento de complexos ciclos biológicos, múltiplas fases de doenças, diferentes morfologias de um mesmo parasita em fases distintas de vida. Ou seja, a Parasitologia é uma disciplina com muitos aspectos visuais para compreensão, que, muitas vezes, não são viáveis para demonstração numa atividade prática real, sendo assim, é um campo fértil para a aplicação das TDICs.

Nessa perspectiva, o objetivo desse estudo foi descrever as produções científicas que relatam o uso de tecnologias digitais com vistas a promover e aperfeiçoar o ensino da Parasitologia no ambiente educacional.

\section{Métodos}

Esse estudo é uma revisão bibliográfica integrativa. Nessa estratégia, realiza-se uma análise da literatura sobre temática específica, visando fornecer uma integração de resultados e também propor lacunas para novos estudos ${ }^{7}$. Foram selecionados estudos descritivos, observacionais, incluindo artigos, monografias, dissertações e teses que versavam sobre o tema de ensino de Parasitologia com a utilização de tecnologias digitais como ferramentas de apoio ao aprendizado, publicadas nos idiomas português e espanhol, 
no período compreendido entre 2008 e 2019. Salienta-se que a delimitação desse período se deu em razão de ser o período em que tais tecnologias haviam sido incorporadas na rotina dos indivíduos e estavam disponíveis para o uso da comunidade acadêmica, portanto, podendo ser direcionadas para o ensino. Para aumentar a capacidade de busca nas diferentes bases de dados foram aplicadas as estratégias descritas a seguir.

Nas bases científicas LILACS e SCIELO foram utilizados os termos descritores "Parasitologia TIC", "Parasitologia Tecnologia(s) Digital(ais)", "Parasitologia Midia(s) Digital(ais)”, “Parasitologia Ensino”, “Parasitologia Herramienta Enseñanza”, “Parasitologia Enseñanza", "Aprendizagem a distancia Parasitologia" e "Aprendizaje a distancia Parasitologia".

Na base Google Academics foi aplicada a estratégia da busca avançada delimitada pelo preenchimento dos critérios: Encontrar artigos com todas as palavras: "--" e com a frase exata: "--". Esses espaços "--" foram preenchidos, respectivamente, pelos termos: "Digital” e "Ensino de Parasitologia”, "Ensino de Parasitologia" e "mídias digitais", "Ensino de Parasitologia" e "Ferramenta de Ensino", “Ensino de Parasitologia" e "Tecnologia Digital”, "Enseñanza de Parasitologia” e "Digital”, "Enseñanza de Parasitologia” e "Herramienta”, "Parasitologia" e "Aprendizagem a distancia" e "Parasitologia" e "Aprendizaje a distancia".

$\mathrm{Na}$ base de dados geral do Google também foi aplicada a estratégia de busca avançada na qual delimitou-se a busca pelo preenchimento dos critérios: Encontrar páginas com todas as palavras: "--" e esta expressão ou frase exata: "--". Nesse caso, os espaços "-" foram preenchidos, respectivamente, pelos termos: "Ensino de Parasitologia" e "TIC", "Ensino de Parasitologia" e "Tecnologia Digital”, "Ensino de Parasitologia" e "Tecnologias Digitas", "Ensino de Parasitologia" e "Mídias digitais", “Ensino de Parasitologia" e "Ferramenta de Ensino".

Para aumentar a abrangência e a possibilidade de triar outros estudos que, eventualmente, não tenham sido recuperados nas buscas sistematizadas em razão da diversidade de termos utilizados na catalogação dos trabalhos na área, foi ainda realizada uma busca complementar na base geral Google contendo as palavras-chave: "Tecnologia digital ensino de parasitologia".

O critério de inclusão foi: trabalho científico completo reportando dados qualitativos ou quantitativos sobre tecnologias digitais e sua aplicação no ensino de Parasitologia. Nesse sentido, incluíram-se artigos em periódicos científicos, artigos completos em eventos científicos, monografias, dissertações e teses. 
Os critérios de exclusão foram: publicação em websites generalistas, propagandas veiculadas em mídias, resumos simples publicados em anais eventos, projetos de pesquisa e extensão. Também foram excluídas outras publicações científicas que apesar de tratar do ensino de Parasitologia por ferramentas diversificadas, tais como jogos de carta, jogos de tabuleiro, pôsteres, atividades de grupo, atividades lúdicas presenciais, não faziam uso de nenhum recurso tecnológico digital (virtual).

Os critérios de inclusão e exclusão foram aplicados ao longo da busca tanto pela leitura dos títulos dos trabalhos, o que permitia descartar logo de início trabalhos fora da temática, quanto pela leitura dos resumos ou do material completo quando se fez necessário. O software livre IHMC CmapsTools v.6.04 foi utilizado para a criação da esquematização da Figura 2.

\section{Resultados}

Da aplicação da estratégia de busca na base científica LILACS foram encontrados 44 documentos, dentre os quais 5 foram selecionados após aplicação dos critérios de inclusão e exclusão. Da aplicação da estratégia na base científica SciELO foram encontrados 25 documentos, dentre os quais 1 foi selecionado após aplicação dos critérios de inclusão e exclusão. Da aplicação da estratégia na base de dados Google Academics foram encontrados 197 documentos, dentre os quais 8 foram selecionados após aplicação dos critérios de inclusão e exclusão. Da aplicação da estratégia na base geral Google foram encontrados 104 documentos, não sendo selecionado nenhum trabalho após a aplicação dos critérios de inclusão e exclusão. Da aplicação da estratégia da busca complementar na base geral do Google foram encontrados 120 documentos, dentre os quais seis (06) foram selecionados após aplicação dos critérios de inclusão e exclusão.

Ressalta-se que, ao longo das buscas, diversos trabalhos eram recuperados de modo repetido. Ao todo, as buscas retornaram repetições, ou seja, trabalhos que haviam sido recuperados por uma ou outras estratégias de buscas. Por exemplo, na busca na base geral Googlehouve o retorno de 6 documentos repetidos que haviam sido selecionados por outras buscas, por isso, nenhum foi selecionado por essa estratégia. Portanto, o conjunto de estratégias aplicado nas buscas permitiu recuperar 490 trabalhos diversos, 44 eram comuns às diferentes estratégias aplicadas nas buscas e 20 foram definitivamente selecionados para compor esse estudo de revisão integrativa. A Figura 1 esquematiza as estratégias e os resultados das buscas. 
Os 20 estudos selecionados foram publicados entre os anos de 2011 a 2019, sendo os anos de 2013 e 2019 aqueles com maior número de trabalhos, 4 em cada ano. Os estudos consistiram de artigos completos em periódicos $(n=11)$, artigos completos em eventos científicos $(n=5)$, monografia de especialização $(n=1)$, dissertação de mestrado $(n=2)$ e tese de doutorado $(n=1)$.

Os públicos alvos dos estudos foram constituídos prioritariamente por estudantes de cursos de graduação na área da saúde, particularmente a medicina, e biológicas $(n=8)$, seguidos de alunos do ensino fundamental $(n=4)$, profissionais técnicos da área da saúde $(n=2)$ e público piloto $(n=2)$ que apenas testaram as ferramentas para validação inicial. Alguns trabalhos $(n=4)$ apenas relataram as estratégias digitais criadas não aplicando em nenhum público para validação. Todos os trabalhos descreveram estratégias de TDICs como ferramenta para auxiliar o ensino de Parasitologia, atendendo ao critério de inclusão dessa revisão integrativa, conforme descrito na Tabela 1.

Um mapa integrado dos recursos digitais e os públicos alvos descritos nos trabalhos pode ser visualizado na esquematização mostrada na Figura 2. No Quadro 1, são elencados e descritos os respectivos objetivos e os principais resultados encontrados nos artigos selecionados, permitindo fazer uma avaliação mais detalhada do teor dos trabalhos.

Os autores propuseram diferentes estratégias, que seguem descritas: 6 trabalhos propuseram sítios eletrônicos (sites) para armazenamento e disponibilização de materiais diversos, 5 trabalhos criaram algum tipo de softwares que poderia ser usado em computadores e aparelhos smartphones (ex. estação de trabalho ligado a um sistema de laboratório à distância), 4 trabalhos implementaram conteúdos da disciplina em Ambientes Virtuais de Aprendizado (AVA), tipo Moodle, 2 trabalhos criaram jogos digitais com temática de Parasitologia, 2 trabalhos criaram Atlas virtuais com funcionalidades diversificadas e 1 trabalho, mais recente publicado em 2019, criou um aplicativo de smartphones para disponibilizar conteúdos para estudo do protozoário Toxoplasma gondii. Todos os produtos eram destinados ao processo de ensino e aprendizagem da temática Parasitologia e seus aspectos, tais como, morfologia, ciclo biológico, patogenia, diagnóstico, métodos de prevenção, que, de um modo geral, amplia a abordagem às doenças parasitárias.

Pode-se observar que as justificativas para as criações das estratégias eram essencialmente baseadas em poder auxiliar os estudantes à melhor compreensão dos aspectos e conteúdos da parasitologia, que têm muitos conceitos visuais, ciclos, morfologias distintas para em um mesmo organismo em diferentes fases da vida. Isso 
corrobora com o que foi citado anteriormente sobre as funcionalidades que as estratégias digitais trazem ao ensino dessa área. Os métodos visuais, por exemplo, por meio de atlas, são estratégias bastante eficientes no ensino de parasitologia ${ }^{5}$. Por isso, essa é uma disciplina frequente e tradicionalmente acompanhada por atlas de imagens, mesmo na modalidade em papel, para apoio ao aprendizado. Nesse sentido, os recursos digitais tornam o processo de aprendizado mais dinâmico, proporcionando uma maior interatividade visual, e também reduzindo os altos custos envolvidos na produção em papel de imagens em alta resolução 8 .

Os resultados encontrados ressaltaram a importância do tema "parasitologia" não apenas para o ensino superior, como também para o ensino fundamental e técnicoprofissionalizante. 0 estudo da Parasitologia permanece um tema atemporal, particularmente no Brasil que, devido às desigualdades sociais, ainda tem uma parcela da população muito atingida por parasitoses, especialmente as intestinais. Entretanto, para aumentar a capacidade de conscientização e prevenção às infecções parasitárias, é necessário ultrapassar a barreira do ensino tradicional, atingindo o público alvo com estratégias que atraiam maior atenção ao conteúdo ${ }^{9}$. Nesse sentido, a utilização das TDICs pode melhorar o processo de ensino e aprendizagem.

A modernização do cenário da ciência, invariavelmente, passa pela construção de bases virtuais de estudo e treinamento ${ }^{10}$, como pode ser visto no uso de conhecidos ambientes virtuais de aprendizagem, tal como o Moodle ${ }^{11}$, para alunos na disciplina de Parasitologia. Ressalta-se que as TDICs, que também podem ser consideradas ferramentas de metodologias ativas, propiciam o maior protagonismo do estudante em seu aprendizado, pois ele tem que interagir com as ferramentas para aumentar seu nível de conhecimento e, com isso, ele é estimulado a pensar e criar uma visão crítica dos conteúdos aprendidos ${ }^{12}$. 0 ensino com apoio virtual tem grande aceitação pelo alunado, é apoiada pela comunidade científica $^{1}$ e utilizada na capacitação de outros profissionais da saúde ${ }^{13}$, sendo inclusive bastante funcional nas situações em que a formação tem que ser realizada à distância ${ }^{14}$.

A Parasitologia é um tema que, além de ser conteúdo obrigatório de muitas carreiras nas áreas das biociências ${ }^{15}$, contemplado em disciplinas de Parasitologia (básica, humana, médica ou clínica) para os cursos da Graduação, também é importante para os alunos do ensino fundamental ${ }^{16,17}$. Segundo os professores desse nível educacional, os conceitos de Parasitologia fazem parte dos seus cotidianos e das suas famílias. Entretanto, os conteúdos dessa temática na graduação têm forte abordagem medicalizante, e são ensinados de modo 
pouco contextualizado e problematizado considerando o amplo espectro das doenças parasitárias ${ }^{18}$. Ainda assim, observou-se que os professores que participaram da validação das estratégias relataram conhecimentos prévios a respeito do tema, ou seja, em algum momento tiveram contato com o assunto. Considerando o público de estudantes no ensino fundamental, tem-se que o desenvolvimento de jogos e materiais digitais, a fim de utilizarse da ludicidade para aumentar o nível de aprendizado e aprofundamento dos conceitos abordados na Parasitologia, são essenciais, reiterando a necessidade do compartilhamento desses saberes o mais precocemente possível ${ }^{19}$.

Indo de encontro aos resultados descritos, percebeu-se ainda a importância do treinamento dos profissionais agentes de saúde na temática proposta ${ }^{20}$. Essa formação contínua que as plataformas digitais propiciam é especialmente importante para esses profissionais, devido a realidade das doenças parasitárias no cotidiano dos indivíduos residentes em uma comunidade. Além disso, as inúmeras possibilidades que as TDICs, como ferramenta de transmissão do saber a distância, oferecem para atingir esse público, ajuda a transpor as barreiras geográficas e de infraestrutura.

\section{Conclusão}

O tema "Parasitologia e Doenças Parasitárias" permanece atual, mantendo a pertinência da sua abordagem desde o ensino fundamental, passando pelo ensino técnico e imprescindível nos cursos de graduação nas áreas da saúde e biológicas. Devido a importância que a discussão sobre prevenção de doenças assumiu nos dias atuais, o uso das TDICs encontra cada vez mais aplicações na remodelagem do ensino tradicional em um ensino integrado, interativo e atualizado. Nesse contexto, as TDICs têm se tornado ferramentas regulares no processo da aprendizagem de Parasitologia e suas nuances em qualquer dos níveis hierárquicos educacionais, pois seus conhecimentos se aplicam à saúde de todo indivíduo.

Verificou-se que o uso das tecnologias digitais vem aumentando sua presença no ensino de Parasitologia, porém estudos sistemáticos que mostrem sua eficácia na área são encontrados ainda em números reduzidos. A aplicação de tecnologias digitais na área de Parasitologia ainda envolve poucas estratégias e, basicamente, dá-se pelo uso de ambientes virtuais de aprendizagem (AVA), tal como o Moodle, criação de sítios eletrônicos para armazenamento de conteúdos visuais e textuais (sites) e o desenvolvimento de materiais digitais destinados a facilitar a absorção de conceitos, tais como jogos e atlas virtuais. 
Entretanto, são muitas as possibilidades existentes a serem exploradas e criar interatividades no ensino de Parasitologia, por exemplo, ambientes virtuais que simulem a realidade dos ambientes sociais e suas interações, jogos de perguntas e respostas pontuadas e baseadas em textos científicos, mapas conceituais virtuais e histórias em quadrinhos são alguns exemplos dentre as múltiplas possibilidades presentes nas TDICs.

Portanto, é imprescindível a manutenção dessas iniciativas, não apenas no desenvolvimento, mas também na divulgação constante de novos materiais e resultados de aplicações experimentais que complementem a área de ensino de Parasitologia, haja vista sua grande importância em saúde pública e coletiva.

\section{Agradecimentos}

Os autores agradecem a Universidade Federal de São Paulo e ao curso de Especialização de Informática em Saúde.

Conflito de interesse: os autores relatam que não há conflito de interesse.

\section{Referências}

1. Palácio MAV, Struchiner M. Análise do uso de recursos de interação, colaboração e autoria em um ambiente virtual de aprendizagem para o ensino superior na área da saúde. Ciência \& Educação (Bauru) 2016, 22(2): 413-430. Disponível em <http://dx.doi.org/10.1590/1516-731320160020009>.

2. Souza CS, Iglesias AG, Pazin-Filho A. Estratégias inovadoras de ensino. Medicina (Ribeirão Preto) 2014, 47(3): 284-92. Disponível em < http://www.revistas.usp.br/rmrp/article/view/86617>.

3. Bechara, JJB. Aprendizagem em ambientes virtuais: estamos utilizando as pedagogias mais adequadas? 2006. 102 f. Dissertação (Mestrado) - Programa de Pós-graduação em Educação, Universidade Federal do Rio de Janeiro (UFRJ), Rio de Janeiro, 2006. Disponível em < http://www.latec.ufrj.br/monografias/2006_Joao_Bechara.pdf>.

4. Farias $L A B G$, Sousa RB, Almeida JBCP, Silveira T, Sousa CL, Teixeira MJ. A utilização de mídias digitais como ferramenta difusora de conhecimento e informação na disciplina de Parasitologia. In: Anais do Encontro de Iniciação à Docência. 2016, Fortaleza. Fortaleza: UFC, 2016, 1(1): 2417-2417. Disponível em <http://periodicos.ufc.br/eu/article/view/16346>.

5. Franco AR, Araújo IL, Carvalho PG. Elaboração de um atlas para o estudo da Parasitologia como estratégia de aprendizagem. In: Anais do Encontro De Iniciação à Docência, Fortaleza. Fortaleza: UNIFOR, 2017, 1-6. Disponível em <https://uol.unifor.br/oul/conteudosite/?cdConteudo=7914404>.

6. Silveira T, Almeida JBCP, Melo ACLF. Uso de mídias digitais e sociais na disponibilização de material de apoio ao ensino de Parasitologia em cursos da área da saúde. In: Anais do II Encontro de tecnodocência 2016, Encontros Universitários 
da UFC, Fortaleza. Fortaleza: UFC, 2016, 1: 4853-4853. Disponível em <http://repositorio.ufc.br/handle/riufc/2675>.

7. Mendes KDS, Silveira RCCP, Galvão CM. Revisão Integrativa: Método de Pesquisa para incorporação de evidências na Saúde e na Enfermagem. Texto Contexto Enferm, Florianópolis, 2008, 17(4): 758-64. Disponível em <http://dx.doi.org/10.1590/S0104-07072008000400018>.

8. Bahia NS et al. O uso das TDIC's como estratégia para aprendizagem em morfologia microscópica. Informática na Educação: teoria \& prática, 2019, 22(2): 110-124. Disponível em <https://seer.ufrgs.br/InfEducTeoriaPratica/article/view/88412>.

9. Costa IG, Paula IL, Gontijo LM, Rocha SR, Costa FJ. Intervenções educativas sobre parasitologia no ensino fundamental: a necessidade de inserir novas metodologias. Revista Tecer, 2017, 10(18):54-63.

10. Cornelio OM, Rubido MBC. Práctica de Microbiología y Parasitología Médica integrado al Sistema de Laboratorios a Distancia en la carrera de Medicina. Revista Ciencias Médicas de Pinar del Río 2016, 20(2): 174-181. Disponível em <http://scielo.sld.cu/scielo.php?script=sci_arttext\&pid=S156131942016000200005>.

11. Mezzari A. O Uso do Moodle como Reforço ao Ensino Presencial de Parasitologia e Micologia no Curso de Graduação em Medicina. Revista Brasileira de Educação Médica 2012, 36(4): 557-563. Disponível em <http://dx.doi.org/10.1590/S0100$55022012000600016>$.

12. Miranda AP. Análise crítica-reflexiva na utilização da metodologia ativa. Enfermagem Brasil 2017;16(3):182-189. Disponível em $<$ https://portalatlanticaeditora.com.br/index.php/enfermagembrasil/article/view/11 $53 / 2276>$.

13. Domenico EBL, Cohrs CR. Plataforma Moodle na construção do conhecimento em Terapia Intensiva: estudo experimental. Acta Paulista de Enfermagem 2016, 29(4): 381-389. Disponível em <http://dx.doi.org/10.1590/1982-0194201600053>.

14. Benelli JL, Gil LS. Aceitação de metodologias de ensino à distância na área da saúde: Uma revisão integrativa. Revista Brasileira de Educação e Sáude (REBES) 2018, 8(1):7-17. Disponível em <https://gvaa.com.br/revista/index.php/REBES/article/view/5533>.

15. Andrade AF, Madeira CAG, Melo HHARF. Batalha de Vetores Virtual: uma proposta de jogo pedagógico para o ensino de biociências. In: Anais do Congresso Internacional de Informática Educativa TISE, Nuevas Ideas en Informática Educativa, 2013, Porto Alegre: UFRGS, 2013, 105-112. Disponível em <http://www.tise.cl/volumen9/TISE2013/105-112.pdf>.

16. Silva PCP, Jesus $M H$, Cordeiro JC. A utilização de jogos digitais como recurso de ensino aprendizado das parasitoses humanas no ensino fundamental II. In: Anais do ENID 2013, Areia. Areia: UFPB, 1-7. Disponível em <http://www.prac.ufpb.br/enex/XVENID/PROLICEN/CE/37.docx>.

17. Dias CAC, Kovaliczn RA. Parasitoses humanas e o uso de recursos midiáticos na aprendizagem. Cadernos PDE 2014, 1:1-19. Disponível em <http://www.diaadiaeducacao.pr.gov.br/portals/cadernospde/pdebusca/producoe s_pde/2014/2014_uepg_cien_artigo_carlos_alberto_de_carvalho_dias.pdf>.

18. ODA W, Delizoicov D. Docência no Ensino Superior: as disciplinas Parasitologia e Microbiologia na formação de professores de Biologia. Revista Brasileira de Pesquisa em Educação em Ciências, 2011, 11(3): 101-121. 
19. Costa, ECP. Ação dialógica e comunicativa como referenciais para o ensino de enteroparasitoses: possibilidades e desafios no Ensino Fundamental. Tese (Doutorado). Programa de Doutorado em Ensino em Biociências e Saúde, FIOCRUZ, Rio de Janeiro, 2017, 244 f. Disponível em <https://www.arca.fiocruz.br/xmlui/handle/icict/25164>.

20. Ferreira GR. Capacitação por ensino à distância de agentes de saúde na prevenção de doenças parasitárias. Tese (Doutorado) Programa de Biologia Animal, Universidade Estadual de Campinas (UNICAMP), Campinas, 2013, 98 f. Disponível em <http://repositorio.unicamp.br/jspui/handle/REPOSIP/317798>.

21. Mezzari A. O Uso da Aprendizagem Baseada em Problemas (ABP) como Reforço ao Ensino Presencial Utilizando o Ambiente de Aprendizagem Moodle. Revista Brasileira de Educação Médica, 2011, 35(1): 114-121. Disponível em <https://doi.org/10.1590/S0100-55022011000100016>.

22. Carvalho Junior VM, Rolando RFR, Rolando RGR. Abordando Conceitos de Parasitologia com uso de Recursos Midáticos no Ensino Médio. In: VI Encontro Regional de Ensino de Biologia da Regional 2 RJ/ES CEFET/RJ, 2012. Disponível em $<$ http://docplayer.com.br/55378321-Abordando-conceitos-de-parasitologia-comuso-de-recursos-midiaticos-no-ensino-medio.html>.

23. Ortíz EB. Enseñanza de la parasitología veterinaria a partir del uso de organismos vivos y tecnologías de la información y de la comunicación (TIC). Revista de Medicina Veterinária 2012, 23: 97-109. Disponível em <http://www.scielo.org.co/scielo.php?script=sci_arttext\&pid=S0122$93542012000100010 \& \operatorname{lng}=e n \& n r m=i s o \& t \operatorname{lng}=e s>$.

24. Silva WT. Elaboração de um Atlas Virtual de Parasitologia e aplicação no Curso Técnico de Patologia Clínica. 2015. 99 f. Dissertação (Mestrado em Educação nas Profissões da Saúde) - Programa de Estudos Pós-Graduados em Educação nas Profissões da Saúde, Pontifícia Universidade Católica de São Paulo, Sorocaba, 2015. Disponível em <https://tede2.pucsp.br/handle/handle/951>.

25. Ferreira LP, Marques VG, Silva BS, Gama RA. Exercícios Online de Parasitologia e Entomologia. In: Anais do Congresso Regional sobre Tecnologias na Educação, Natal, Brasil, 2016. Disponível em <http://ceur-ws.org/Vol1667/CtrlE_2016_MS_paper_3.pdf>.

26. Marques VG, Silva BS, Gama RA. Atlas Virtual de Parasitologia e Entomologia. In: Anais do Congresso Regional sobre Tecnologias na Educação, Natal, Brasil, 2016. Disponível em <http://ceur-ws.org/Vol-1667/CtrlE_2016_AC_paper_4.pdf>.

27. Archelli SM. Propuesta de incorporación de tecnología digital para los trabajos prácticos a campo, de la materia Parasitología Comparada. Trabajo de especializacion, Facultad de Ciencias Veterinarias, Universidad Nacional de La Plata, Buenos Aires, Argentina, 2017. Disponível em <http://sedici.unlp.edu.ar/handle/10915/62329>.

28. Carballosa YC, Pérez XC, Rojas LT. MicrobiologíaSoft, entrenador de Microbiología y Parasitología médica. Revista Cubana de Informática Médica, 2017, 9(1): 61-72. Disponível em <http://scielo.sld.cu/scielo.php?script=sci_arttext\&pid=S168418592017000100007>.

29. Oliveira RB, Barbin ICC, Silva HS, Curado ACC, Thomaz MCA. Metodologias Ativas de Ensino-Aprendizagem na Formação Profissional em Saúde: Construção de Livro Didático Digital Utilizando a Sala de Aula Invertida e a Problematização. In: Anais do III Congresso de Inovação e Metodologias no Ensino Superior, Belo Horizonte, Minas Gerais 2017. Disponível em <https://congressos.ufmg.br/index.php/congressogiz/CIM/paper/view/687/258>. 
30. Senna KN, Caldeira AMA. Desenvolvimento e Avaliação de um Objeto de Aprendizagem para o Ensino de Parasitologia. In: Anais do $3^{\circ}$ Encontro Nacional de Jogos e Atividades Lúdicas no Ensino de Química, Física e Biologia (Jalequim Level III), UNILA, Foz do Iguaçu, Paraná, 2018. Disponível em $<$ https://dspace.unila.edu.br/123456789/5773>.

31. Aranha VL. Utilização de uma ferramenta digital para o ensino de parasitologia no ensino superior. 2019. 90 f. Dissertação (Mestrado em Ensino de Ciências) Universidade Cruzeiro do Sul, São Paulo, 2019. Disponível em <https://repositorio.cruzeirodosul.edu.br/handle/123456789/361>.

32. Castro NC, Moreira TR. Avaliação do uso de uma plataforma virtual de aprendizagem como estratégia inovadora no ensino da Parasitologia Humana. In: Tecnologias aplicadas aos processos de ensino e aprendizagem / Vinícius Catão de Assis Souza, Juliana Vieira Afonso Magalhães 2019 (organização). Viçosa, MG: UFV, CEAD, livro eletrônico 2019. Disponível em <https://www2.cead.ufv.br/serieconhecimento/wpcontent/uploads/2019/04/Tecnologias-aplicadas-aos-processos-de-ensino-eaprendizagem.pdf>.

33. Rivera-Fernandez N, García-Dávila P, Hernandez AA. Las aplicaciones digitales como herramienta didáctica para el estudio de la Parasitología Médica. Investigación em Educación Médica, 2019 8(31): 64-71. Disponível em <http://dx.doi.org/10.22201/facmed.20075057e.2019.31.18121>.

34. Senna KN, Caldeira AMA. Avaliação de um Objeto de Aprendizagem para o ensino de Biologia à Educação Básica. Revista de Estudos e Pesquisas sobre Ensino Tecnológico (EDUCITEC), 2019, 5(10): 311-328. Disponível em <https://doi.org/10.31417/educitec.v5i10.520>. 


\section{Anexos}

Tabela 1. Artigos selecionados para compor esse estudo de revisão bibliográfica integrativa por ordem de ano da publicação.

\begin{tabular}{|c|c|c|c|c|}
\hline Ano & Autores & Público-alvo & $\mathrm{TIC}$ & $\begin{array}{c}\text { Tipo de } \\
\text { publicação }\end{array}$ \\
\hline 2011 & Mezzari A. ${ }^{21}$ & Alunos do curso de Medicina & ABP* via Moodle & Artigo \\
\hline 2012 & $\begin{array}{c}\text { Carvalho Junior VM, Rolando } \\
\text { RFR, Rolando RGR. }{ }^{22}\end{array}$ & $\begin{array}{l}\text { Alunos do } 2^{\circ} \text { e } 3^{\circ} \text { ano do } \\
\text { ensino médio }\end{array}$ & $\begin{array}{l}\text { Repositório } \\
\text { virtual }\end{array}$ & $\begin{array}{l}\text { Artigo em } \\
\text { evento }\end{array}$ \\
\hline 2012 & $\begin{array}{c}\text { Mezzari A, Iser I, Wiebbelling } \\
\text { AMP, Tarouco L. }{ }^{11}\end{array}$ & Alunos do Curso de Medicina & Moodle & Artigo \\
\hline 2013 & $\begin{array}{c}\text { Andrade AF, Madeira CAG, Melo } \\
\text { HHARF. }{ }^{15}\end{array}$ & $\begin{array}{c}\text { Futuros alunos de Graduação } \\
\text { em biociências }\end{array}$ & Jogo digital & Artigo \\
\hline 2013 & Ferreira GR. ${ }^{20}$ & Agentes de Saúde & $\mathrm{EaD}^{\star \star *} / \mathrm{AVA}^{\star \star *}$ & $\begin{array}{l}\text { Tese de } \\
\text { doutorado }\end{array}$ \\
\hline 2013 & Ortíz EB. ${ }^{23}$ & Não aplicou & $\begin{array}{l}\text { Materiais de } \\
\text { acesso livre na } \\
\text { internet }\end{array}$ & Artigo \\
\hline 2013 & $\begin{array}{c}\text { Silva PCP, Jesus MH, Cordeiro } \\
\text { JC. }{ }^{16}\end{array}$ & $\begin{array}{l}\text { Alunos de } 7^{\circ} \text { ano do ensino } \\
\text { fundamental }\end{array}$ & Jogos digitais & $\begin{array}{l}\text { Artigo em } \\
\text { evento }\end{array}$ \\
\hline 2014 & Dias CAC, Kovaliczn RA. ${ }^{17}$ & $\begin{array}{l}\text { Alunos de } 7^{\circ} \text { e } 8^{\circ} \text { ano do } \\
\text { ensino fundamental }\end{array}$ & $\begin{array}{c}\text { Recursos } \\
\text { midiáticos } \\
\text { virtuais - Pixton }\end{array}$ & Artigo \\
\hline 2015 & Silva WT. ${ }^{24}$ & $\begin{array}{c}\text { Alunos de curso técnico em } \\
\text { patologia clínica }\end{array}$ & Atlas virtual & $\begin{array}{l}\text { Dissertação de } \\
\text { mestrado }\end{array}$ \\
\hline 2016 & Cornelio OM, Rubido MBC. ${ }^{10}$ & Alunos do Curso de Medicina & $\begin{array}{l}\text { Sistema de } \\
\text { Laboratório à } \\
\text { Distância }\end{array}$ & Artigo \\
\hline 2016 & $\begin{array}{c}\text { Ferreira LP, Marques VG, Silva } \\
\text { BS, Gama RA. }{ }^{25}\end{array}$ & Não aplicou & $\begin{array}{l}\text { Exercícios } \\
\text { virtuais }\end{array}$ & $\begin{array}{l}\text { Artigo de } \\
\text { evento }\end{array}$ \\
\hline 2016 & $\begin{array}{c}\text { Marques VG, Silva BS, Gama } \\
\text { RA. }{ }^{26}\end{array}$ & $\begin{array}{c}\text { Aplicação piloto com } \\
\text { professores, monitores e } \\
\text { técnicos }\end{array}$ & Atlas virtual & $\begin{array}{l}\text { Artigo de } \\
\text { evento }\end{array}$ \\
\hline 2017 & Archelli SM. ${ }^{27}$ & Não aplicou & Mural virtual & $\begin{array}{l}\text { Monografia de } \\
\text { especialização }\end{array}$ \\
\hline 2017 & $\begin{array}{c}\text { Carballosa YC, Pérez XC, Rojas } \\
\text { LT. } 28\end{array}$ & $\begin{array}{c}\text { Professores e alunos de curso } \\
\text { de Medicina }\end{array}$ & Software & Artigo \\
\hline 2017 & $\begin{array}{c}\text { Oliveira RB, Barbin ICC, Silva HS, } \\
\text { Curado ACC, Thomaz MCA. }{ }^{29}\end{array}$ & Apenas disponibilizado na IES & $\begin{array}{l}\text { Plataforma web } \\
\text { institucional }\end{array}$ & Artigo \\
\hline 2018 & Senna KN, Caldeira AMA. ${ }^{30}$ & $\begin{array}{c}\text { Validação com licenciandos } \\
\text { de Biologia }\end{array}$ & $\begin{array}{c}\text { Objeto de } \\
\text { Aprendizagem } \\
\text { virtual }\end{array}$ & $\begin{array}{l}\text { Artigo em } \\
\text { evento }\end{array}$ \\
\hline 2019 & Aranha VL. ${ }^{31}$ & $\begin{array}{l}\text { Estudante de graduação nas } \\
\text { áreas da saúde }\end{array}$ & $\begin{array}{c}\text { Ferramenta } \\
\text { digital Powtoon }\end{array}$ & $\begin{array}{l}\text { Dissertação de } \\
\text { mestrado }\end{array}$ \\
\hline 2019 & Castro NC, Moreira TR. ${ }^{32}$ & $\begin{array}{l}\text { Estudantes de graduação em } \\
\text { Enfermagem e Biologia }\end{array}$ & $\mathrm{AVA}^{\star \star \star \star}$ & Artigo \\
\hline 2019 & $\begin{array}{l}\text { Rivera-Fernandez N, García- } \\
\text { Dávila P, Hernandez AA. }{ }^{33}\end{array}$ & $\begin{array}{l}\text { Estudantes de Medicina e } \\
\text { Bolsistas de Departamento de } \\
\text { realidade virtual }\end{array}$ & Aplicativo (app) & Artigo \\
\hline 2019 & Senna KN, Caldeira AMA. ${ }^{34}$ & $\begin{array}{l}\text { Alunos de } 7^{\circ} \text { ano do ensino } \\
\text { fundamental }\end{array}$ & $\begin{array}{c}\text { Objeto de } \\
\text { Aprendizagem } \\
\text { virtual }\end{array}$ & Artigo \\
\hline
\end{tabular}

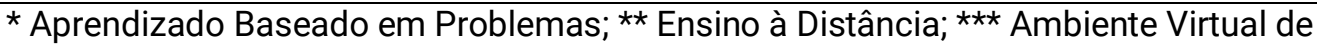
Aprendizagem.

Fonte: Elaborado pelos autores. 
Figura 1. Esquematização das estratégias de buscas e seleção dos trabalhos realizados para essa revisão bibliográfica integrativa.

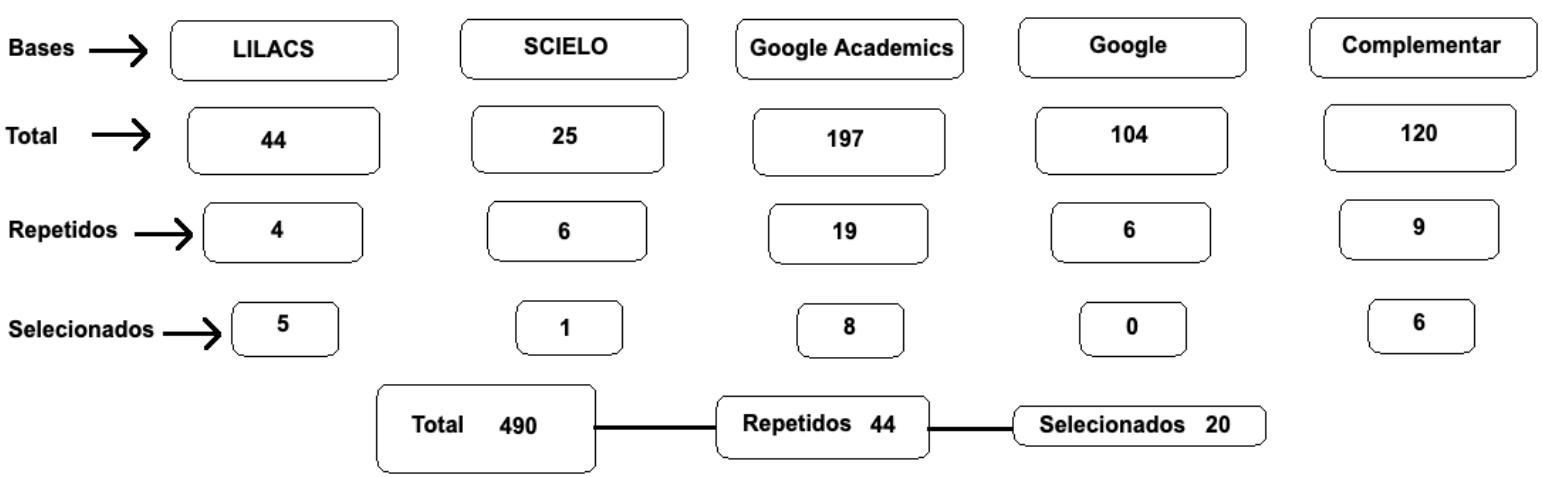

Fonte: elaborado pelos autores.

Figura 2. Esquematização dos recursos de tecnologias digitais e os públicos alvos pretendidos conforme descrição nos artigos selecionados para compor esse estudo de revisão bibliográfica integrativa.

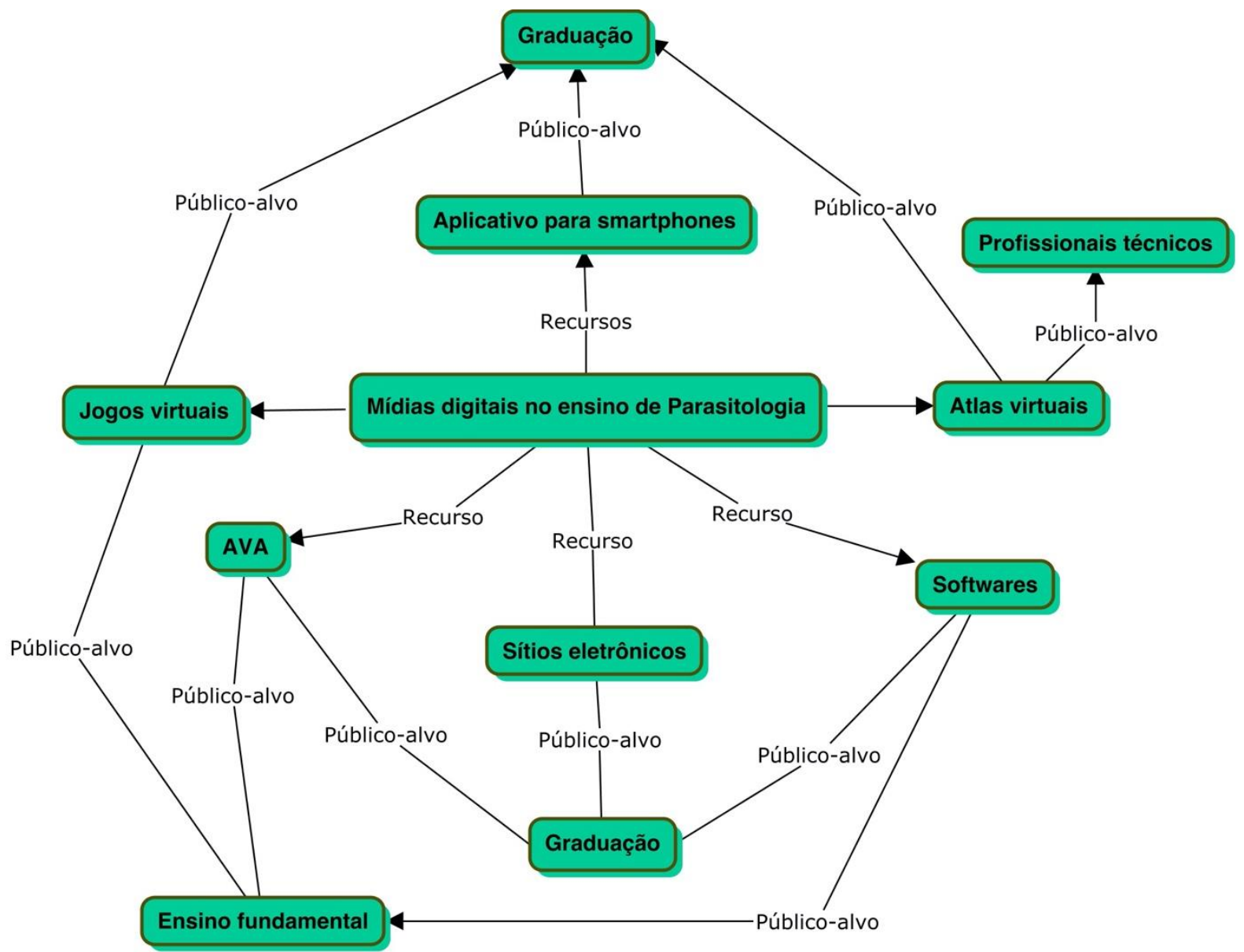

Fonte: elaborado pelos autores com uso do software livre CmapsTools v.6.04. 